HNO 2021 $69: 650-657$

https://doi.org/10.1007/s00106-021-01050-z

Angenommen: 20. Januar 2021

Online publiziert: 14. April 2021

(c) Der/die Autor(en) 2021
C. Plettenberg 1 - K. Geipel • I. Stenin · T. Klenzner · M. Wagenmann · J. Schipper • K. Scheckenbach

Hals-Nasen-Ohrenklinik, Zentrum für Operative Medizin II, Universitätsklinikum Düsseldorf, Düsseldorf, Deutschland

\section{Tröpfchenexposition bei Tracheotomie}

\section{Fallanalyse und Konsequenzen in Bezug auf COVID-19-Patienten}

\section{Hintergrund}

Aufgrund der hohen Infektiosität von COVID-19 („,coronavirus disease 2019“) und gleichzeitiger hoher Virusdichte in den oberen Atemwegen müssen Tracheotomien, ein tröpfchen- und aerosolerzeuziell infektiöser Eingriff gelten. Zur Vermeidung von Infektionen und eventuellen schweren Krankheitsverläufen bei medizinischem Personal ist es wichtig, das Infektionsrisiko zu kennen und entsprechende Schutzmaßnahmen auf dieser Grundlage einzuführen.

\section{Fragestellung}

Das SARS-CoV-2-Virus, aus der Gruppe der Coronaviridae, zeichnet sich durch eine hohe Infektiosität und eine Übertragung durch Tröpfcheninfektionen aus. Eine sehr hohe Viruslast wurde im Sekret der oberen Atemwege (Nase/Rachen) detektiert [19]. Zu Risikoeingriffen bei diesen Patienten gehören deshalb Untersuchungen und Eingriffe an den oberen Atemwegen wie Bronchoskopien, Intubationen und Tracheotomien. Eine besondere Exposition von Mitarbeitern im Gesundheitswesen, die vermutlich mit einer erhöhten Rate an Erkrankungen einhergeht, ist im Rahmen der aktuellen Pandemie beschrieben worden [25, 37]. Obwohl die Infektion in den meisten Fällen milde verläuft, gibt es lebensbedrohliche Krankheitsverläufe. Diese zeichnen sich vor allem durch den Übergang der Erkrankung in ein schweres gender medizinischer Eingriff, als poten- akutes respiratorisches Syndrom (SARS) aus. Die Patienten werden schnell beatmungspflichtig, und im Fall einer Langzeitbeatmung wird eine Tracheotomie zum besseren Atemwegsmanagement, zur Vermeidung von intubationsbedingten Komplikationen und eventuell zur Verbesserung des Outcomes angestrebt. Für den HNO-Arzt steht die Tracheotomie bei COVID-19-Patienten deshalb in einem besonderen Fokus, da es bei der Eröffnung der Trachea regelhaft zu einer Verteilung von Tracheal- und Bronchialflüssigkeiten im Op.-Gebiet und seiner Umgebung kommt. Gleichzeitig findet sich eine besonders hohe Konzentration des Virus in den oberen Atemwegen [39]. Zusätzlich ist bekannt, dass sich SARS-CoV-2 je nach Oberflächenma- terial und -beschaffenheit bis $\mathrm{zu} 72 \mathrm{~h}$ nachweisen lässt [12]. Bereits bei der SARS-Epidemie 2004 zeigte sich, dass insbesondere das medizinische Personal durch seine Patientenexposition gefährdet ist. So waren z. B. in Hongkong 400 von 1755 SARS-Patienten Beschäftigte im Gesundheitswesen [3]. Sorgfältige Beachtung der Infektionskontrolle und adäquater Infektionsschutz sind deshalb unerlässlich, um Übertragungen des Virus vom Patienten auf Mitarbeiter zu minimieren. Die Dringlichkeit zur Prävention im Vergleich zu anderen Viren ergibt sich aufgrund der hohen Infektiosität und der hohen Transmissionsraten [16]. Um einen Eindruck über die Tröpfchenexposition, -verteilung und -art zu bekommen, wurden bei nichtinfektiösen

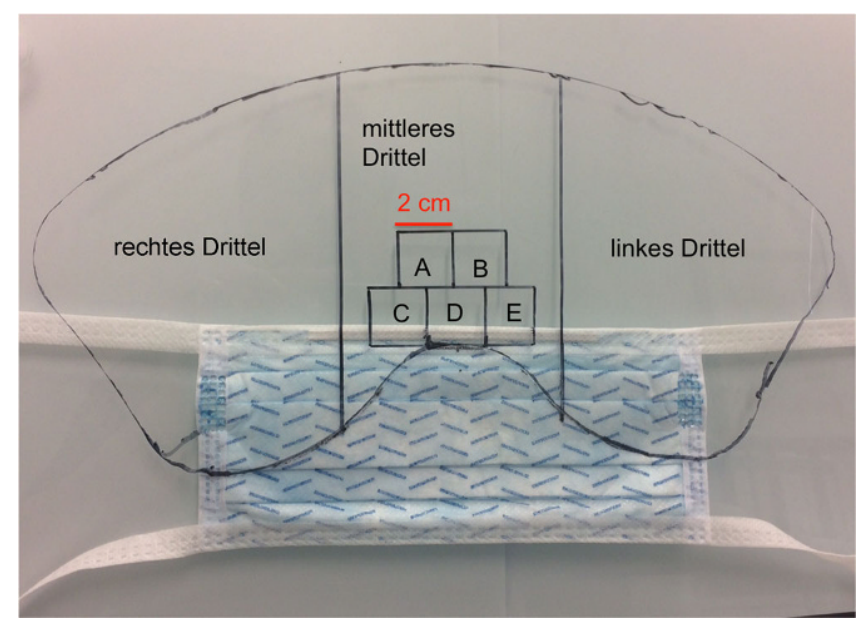

Abb. $1 \Delta$ Sentinex Op.-Maske Fluid Shield ${ }^{\circledR}$ (Fa. Lohmann und Rauscher, Neuwied, Deutschland) mit Unterteilung in mittleres, rechtes und linkes Drittel und Umrandung des gesamten Plexiglases zur besseren Visualisierung, der zentrale Gesichtsbereich wurde zur Auswertung in 5 Quadrate ( $A$ bis $E$ ) von $2 \mathrm{~cm}$ Kantenlänge unterteilt 

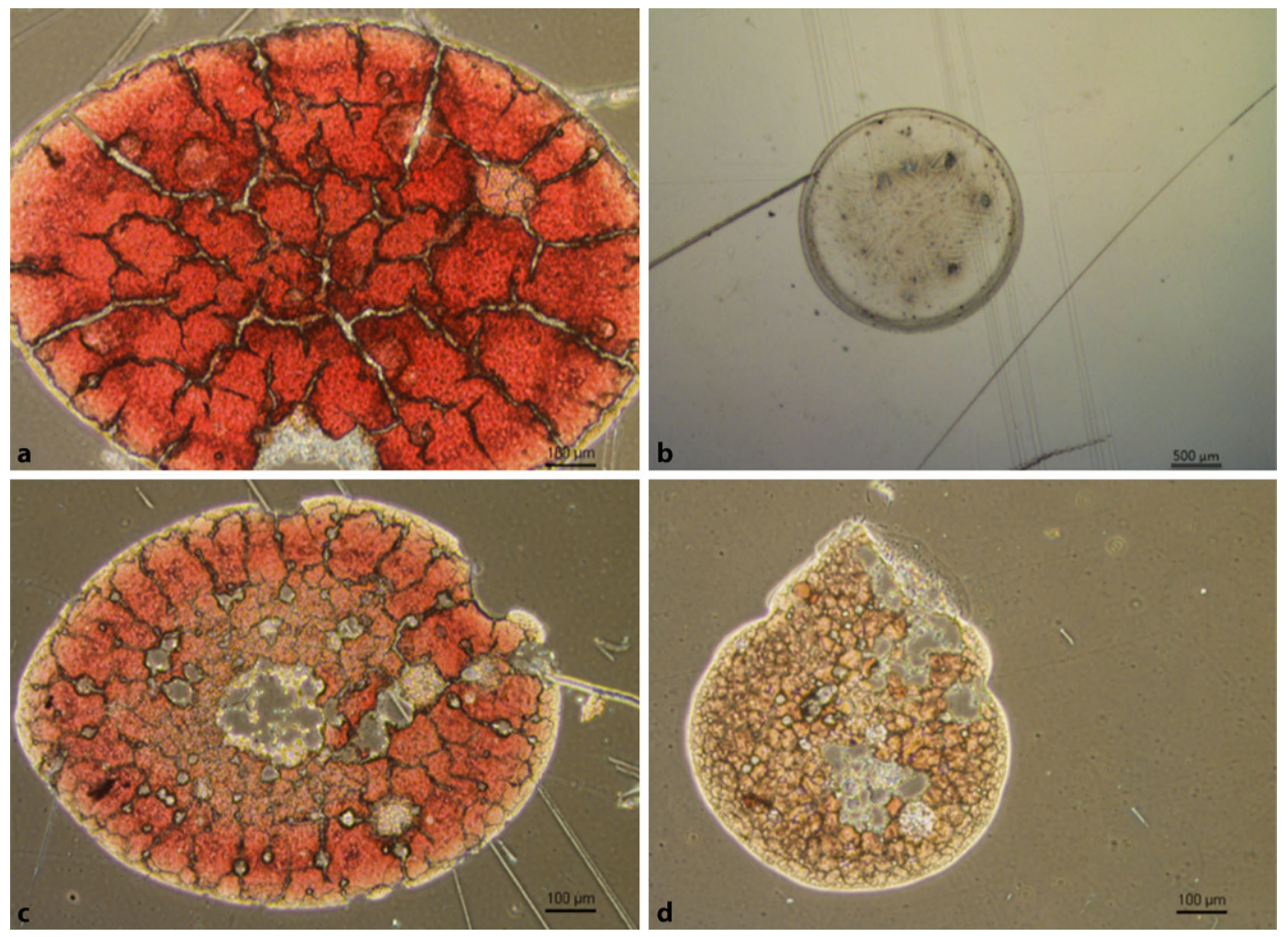

Abb. $2 \triangleleft$ Mikroskopische Darstellung einzelner Tropfen, a rein rote Tropfen (Blut), b rein transparente Tropfen (Sekret), c, d gemischte Tropfen (Blut und Sekret)

Patienten die Visiere des verwendeten Mund-Nasen-Schutzes mit integriertem Visier untersucht.

\section{Material und Methoden}

\section{Operatives Setting und Asservation der Schutzmasken}

Es wurden 4 langzeitbeatmete nichtinfektiöse Patienten chirurgisch von je 2 Operateuren tracheotomiert. Alle Beteiligten hatten einen Abstand von $30-80 \mathrm{~cm}$ vom Operationsgebiet und waren der direkten perioperativen Umgebung ausgesetzt. Während der Prozedur trugen die beiden Operateure und die Assistenz eine Mund-NasenMaske mit integriertem Gesichtsschutz (Sentinex Op.-Maske Fluid Shield der Fa. Lohmann und Rauscher [Neuwied, Deutschland]; - Abb. 1). Diese $(n=8)$ wurden nach Beendigung der Operation ohne Oberflächenkontakt bei Erhalt der postoperativen Oberflächenbeschaffenheit anonym asserviert, für mindestens $24 \mathrm{~h}$ getrocknet und an diesem Material unter Durchleuchtung eine Analyse von Tröpfchenanzahl und -beschaffenheit durchgeführt.
Zur Analyse wurden die Masken in 3 Teilbereiche (frontal, lateral rechts, lateral links) mit je gleicher Breite aufgeteilt (๑Abb. 1). In Rücksprache mit der hiesigen Ethikkommission ist für die patientenunabhängige Materialanalyse der Masken keine Einwilligung des Patienten notwendig. Alle beschriebenen Untersuchungen wurden in Kooperation mit der zuständigen Ethikkommission, im Einklang mit nationalem Recht sowie gemäß der Deklaration von Helsinki von 1975 (in der aktuellen, überarbeiteten Fassung) durchgeführt.

\section{Messung der Tröpfchendichte und -qualität}

Es erfolgte die makroskopische Auszählung der gesamten Maskenfläche. Der Bereich oberhalb der Nase wurde in 5 Areale von je $2 \times 2 \mathrm{~cm}$ Größe eingeteilt und zusätzlich mikroskopisch in 2,6facher Vergrößerung ausgezählt. In einem repräsentativen Areal, $20 \mathrm{~cm}^{2}$ im zentralen Bereich um Nase und Auge jeder Maske, wurde in 4- und in 10facher Vergrößerung mikroskopisch die Zusammensetzung der Tröpfchen (Sekret/Blut) und die Größe der Tröpfchen analysiert. Hierbei wurden je Feld die Tröpfchen anhand einer im Mikroskop integrierten Skala ausgemessen. Außerdem erfolgte eine Fotodokumentation der Befunde (• Abb. 2).

\section{Statistische Auswertung}

Für die Tröpfchenverteilung und -größe wurden Mittelwerte und Standardabweichungen sowie die Odds Ratio arealbezogen berechnet.

\section{Ergebnisse}

\section{Auswertung der Tracheotomien}

Bei insgesamt 4 Tracheotomien konnten 8 Gesichtsschilde ausgewertet werden. Es liefen 3 Eingriffe regelrecht ohne Komplikationen ab. Bei einem Eingriff kam es intraoperativ durch Schlitzung mit dem Skalpell zu einem Defekt des Tubus-Cuffs ohne weitere negative Auswirkungen auf den Operationsverlauf. Die Visiere des Eingriffs wurden jedoch zusätzlich gesondert betrachtet. 
HNO 2021 • 69:650-657 https://doi.org/10.1007/s00106-021-01050-z

(c) Der/die Autor(en) 2021

\section{Plettenberg · K. Geipel · I. Stenin · T. Klenzner · M. Wagenmann · J. Schipper · K. Scheckenbach}

\section{Tröpfchenexposition bei Tracheotomie. Fallanalyse und Konsequenzen in Bezug auf COVID-19- Patienten}

\section{Zusammenfassung}

Hintergrund. Die Pandemie COVID-19

("coronavirus disease 2019") zeichnet sich durch eine hohe Infektiosität bei Tröpfchenübertragung und hoher Virusdichte in den oberen Atemwegen aus. Schwere Krankheitsverläufe stehen mit interstitiellen, beatmungspflichtigen Pneumonien in Verbindung, bei denen regelmäßig Tracheotomien (TT), ein tröpfchen- und aerosolerzeugender medizinischer Eingriff, notwendig werden. Die TT als potenzielles Infektionsrisiko für medizinisches Personal wird in der Literatur kaum behandelt. Deshalb war es Ziel dieser Studie, die Tröpfchenexposition des Op.-Teams während der Tracheotomie zu quantifizieren, um hierdurch die Anforderungen an die notwendige persönliche Schutzausrüstung (PSA) besser zu definieren.
Material und Methoden. Bei 4 nichtinfektiösen Patienten wurde eine chirurgische Tracheotomie durchgeführt, bei der der Chirurg und seine Assistenz jeweils eine chirurgische Mund-Nasen-Maske mit Klarsichtvisier trugen. Nach Durchführung des Eingriffs bestimmten wir Tröpfchenart, -verteilung und -menge auf dem Visier makroskopisch und mikroskopisch. Ergebnisse. Auf den Visieren fanden sich durchschnittlich 29 Tröpfchen im mittleren Drittel des Visiers, 4 im rechten Drittel und 13 im linken Drittel, bei einer durchschnittlichen Tröpfchengröße von $571 \mu \mathrm{m}( \pm 381 \mu \mathrm{m})$. Die kleinsten Tröpfchen waren $55 \mu \mathrm{m}$, die größten $1431 \mu \mathrm{m}$ groß. Eine Zunahme der Tröpfchen fanden sich bei vermehrter Ventilation während des Eingriffs. Bluttröpfchen waren häufiger als Sekrettröpfchen.

Schlussfolgerung. Es konnte eine deutliche Kontamination des Gesichtsvisiers mit Tröpfchen dargestellt werden. Gerade im Fall einer TT von hochinfektiösen Patienten, z. B. COVID-19, ist demnach die Verwendung einer Kapuzen-Kopfbedeckung in Kombination mit einem Atemschutzgerät mit Luftreinigung mit Stromversorgung empfehlenswert, um einen Infektionsschutz des Operateurs und der Op.Assistenz bestmöglich zu gewährleisten.

Schlüsselwörter COVID-19 · Pandemie · Tracheotomie · Persönliche Schutzausrüstung · Tröpfcheninfektion

\section{Droplet exposure during tracheotomy. Case analysis and consequences with respect to COVID-19 patients}

\section{Abstract}

Background. The COVID(coronavirus disease)19 pandemic is characterized by high infectivity, droplet transmission, and high viral load in the upper respiratory tract. Severe disease courses are associated with interstitial pneumonia and ventilated patients, in whom tracheotomy (TT) — a droplet- and aerosolproducing medical intervention-is regularly necessary. TT as a potential infection risk for medical staff is scarcely found in the literature. Therefore, the aim of this study was to quantify droplet exposure of the surgical team during $\mathrm{TT}$, to better define the requirements for personal protective equipment (PPE).
Materials and methods. Surgical TT was performed in four non-infectious patients, during which the surgeon and his assistant both wore a surgical nasal mask with a transparent visor. After the procedure, the type, distribution, and number of droplets on the visor were determined macroscopically and microscopically.

Results. An average of 29 droplets were found on the middle third of the visor, 4 on the right third, and 13 on the left third, with an average droplet size of $571 \mu \mathrm{m}( \pm 381 \mu \mathrm{m})$. The smallest droplets were $55 \mu \mathrm{m}$, the largest $1431 \mu \mathrm{m}$. An increase in the number of droplets was found with increased ventilation during the procedure. Blood droplets were more common than secretion droplets. Conclusion. Contamination of the visor with droplets was demonstrated. Especially in the case of TT in highly infectious patients, e.g., COVID-19 patients, the use of hooded headgear in combination with breathing apparatus with air purification and power supply is recommended to ensure best protection from infection for the surgeon and the surgical assistant.

\section{Keywords}

COVID-19 · Pandemic · Tracheotomy · Personal protective equipment $\cdot$ Droplet infection

\section{Auswertung der Tröpfchengröße und -verteilung}

In jedem der 4 Eingriffe kam es zur Tröpfchenübertragung auf das Visier. Die Analyse der Gesichtsschilde ergab Tröpfchengrößen zwischen 55 und $1431 \mu \mathrm{m}$ (•Tab. 1; • Abb. 3), mit einem Mittelwert von $571 \mu \mathrm{m}$ bei einer Standardabweichung von $381 \mu \mathrm{m}$. Die mittlere Anzahl der Tröpfchen betrug im mittleren Drittel des Gesichtsschilds
29,13 Tröpfchen ( $\pm 37,15$ Tröpfchen), im rechten Drittel 4,38 Tröpfchen $( \pm 5,70$ Tröpfchen), im linken Drittel 13,25 Tröpfchen ( $\pm 9,34$ Tröpfchen; - Tab. 2; - Abb.4). Eine Exposition bestand demnach vor allem erwartungsgemäß im mittleren Drittel. Die Expositionswahrscheinlichkeit im mittleren Drittel war demnach 1,65-mal höher als in der Peripherie (Odds Ratio 1,65). Mikroskopisch wurden insgesamt 63 repräsentative Tröpfchen in 4facher und 10facher Ver- größerung ausgewertet. Hierbei fanden sich Tröpfchen mit vermehrten Anteilen von Blut, bei der die zellulären Blutbestandteile, in erster Linie Erythrozyten, gut zur Darstellung kamen (• Abb. 2a), außerdem zeigten sich Tröpfchen ohne zelluläre Bestandteile, die dem austretenden Sekret entsprachen (• Abb. 2b), sowie Tröpfchen mit gemischten Anteilen (•Abb. 2c, d). Das Gesamtverhältnis der Bluttröpfchen zu Sekrettröpfchen war 12,7:1. Bluttröpfchen ließen sich auf 
Tab. 1 Minimale, maximale und mittlere Tröpfchengröße aller Gesichtsschilde $(n=8)$ mit der

Standardabweichung

Minimale Tröpfchengröße

Maximale Tröpfchengröße

$55 \mu \mathrm{m}$

Mittlere Tröpfchengröße ( \pm Standardabweichung)

$1431 \mu \mathrm{m}$

$571 \mu \mathrm{m}( \pm 381 \mu \mathrm{m})$

Tab. 2 Tröpfchenverteilung auf den transparenten Gesichtsschilden.

TT1-4 = Tracheotomien 1-4 mit je 2 Gesichtsschilden $(n=2)$, die Anzahl der Tröpfchen aus den

5 ausgewählten Bereichen im zentralen Feld ( Abb. 1) $=20 \mathrm{~cm}^{2}$

\begin{tabular}{|c|c|c|c|c|c|c|}
\hline & $\begin{array}{l}\text { Gesamtes } \\
\text { Visier } \\
(n=2)\end{array}$ & $\begin{array}{l}\text { Linkes Drittel } \\
(n=2)\end{array}$ & $\begin{array}{l}\text { Rechtes Drittel } \\
(n=2)\end{array}$ & $\begin{array}{l}\text { Mitte } \\
(n=2)\end{array}$ & $20 \mathrm{~cm}^{2}$ & $\begin{array}{l}\text { Mittlere Tröpfchen- } \\
\text { anzahl aller Visiere } \\
\text { (TT1-4) }\end{array}$ \\
\hline \multicolumn{7}{|c|}{ Tröpfchen Blut und Sputum } \\
\hline TT1 & 268 & 54 & 23 & 191 & 54 & 105 \\
\hline TT2 & 37 & 12 & 5 & 20 & 4 & \\
\hline TT3 & 18 & 14 & 1 & 3 & 3 & \\
\hline TT4 & 95 & 26 & 19 & 50 & 18 & \\
\hline \multicolumn{7}{|c|}{ Tröpfchen Blut } \\
\hline TT1 & 260 & 51 & 20 & 189 & 44 & 98 \\
\hline TT2 & 24 & 7 & 5 & 12 & 2 & \\
\hline TT3 & 18 & 14 & 1 & 3 & 3 & \\
\hline TT4 & 90 & 21 & 19 & 50 & 18 & \\
\hline \multicolumn{7}{|c|}{ Tröpfchen Sekret } \\
\hline TT1 & 8 & 3 & 3 & 2 & 0 & 7 \\
\hline TT2 & 15 & 7 & 0 & 8 & 1 & \\
\hline TT3 & 0 & 0 & 0 & 0 & 0 & \\
\hline TT4 & 5 & 5 & 0 & 0 & 0 & \\
\hline
\end{tabular}

allen 8 Shields nachweisen, gemischte oder reine Trachealsekrettropfen nur auf 6 von 8 . Trachealsekrettröpfchen fanden sich 1,8-mal häufiger in der Peripherie als in der Mitte, wobei auch in der Mitte die Verteilung außerhalb des Zentrums war. Bei dem Patienten mit dem defekten Cuff und der Zwischenbeatmung war die Tröpfchenzahl mit 265 auf dem gesamten Shield deutlich höher als bei den anderen Patienten (Mittelwert 50/ Shield). Die Tröpfchengröße bei dem Patienten mit defektem Cuff war kleiner als bei den anderen Patienten, Mittelwert $431 \pm 261 \mu \mathrm{m} \mathrm{zu} 765 \pm 340 \mu \mathrm{m}$, Median $261 \mu \mathrm{m} \mathrm{zu} 857 \mu \mathrm{m}$ (• Abb. 5). In allen tilation zum Tod des Patienten führen kann. Bei limitierten und primär symptomatischen Therapiemöglichkeiten steht hier die suffiziente Beatmung des Patienten im Vordergrund. Langzeitbeatmete Patienten benötigen sehr häufig eine Tracheotomie. Diese reduziert Komplikationen durch den endotrachealen Tubus (laryngeale und tracheale Druckschäden) $[4,26,29]$, verkürzt die Beatmungszeit und verringert das Infektionsrisiko der unteren Atemwege [27]. Außerdem werden das Totraumvolumen, der Atemwiderstand, die Atemarbeit [3, 8, 10, 32, 33, 38] und der Sedierungsbedarf reduziert und Mobilisations- und Kommunikationsmöglichkeiten verbessert [33]. In der AWMF-S1-Leitlinie „Empfehlungen zur intensivmedizinischen Therapie von $\mathrm{Pa}$ tienten mit COVID-19“ sind die Vorund Nachteile einer Tracheotomie, Wahl des Tracheotomiezeitpunkts und der Tracheotomieart zusammengefasst [17].

Aerogene Infektionen, wie SARSCoV-2, aber auch SARS-CoV, MERS$\mathrm{CoV}$ („Middle East respiratory syndrome-related coronavirus") oder Influenzaviren, erfolgen über Aerosole ( $\leq 5 \mu \mathrm{m}$ - Übertragungsweg $>1 \mathrm{~m}$ ) und Tröpfchen $(>5 \mu \mathrm{m}$ - Übertragungsweg $<1 \mathrm{~m})[5,8,14,30]$. Diese werden durch Atmen, Husten, Niesen, Sprechen, Lachen, aber auch bei aerosol- und tröpfchenproduzierenden Untersuchungen oder Eingriffen übertragen. Tröpfchen sedimentieren innerhalb weniger Sekunden, wodurch Tröpfchenkerne entstehen, die als Aerosole in der Luft schweben können [2].

$\mathrm{Zu}$ den Prozeduren im Gesundheitswesen, die Aerosole und Tröpfchen in hohem Maße erzeugen, gehören Bronchoskopien, das Absaugen der Atemwege und die Intubation [5]. Informationen zur Tröpfchenbildung während der Tracheotomie sind bisher rar, obwohl diese perioperativ offensichtlich sind. Thamboo et al. [35] fassten die aktuelle Literatur zur Eingriffen mit Aerosol- oder Tröpfchenbildung zusammen. Als tröpfchenerzeugendes Verfahren erhöht die Tracheotomie die potenzielle Virusexposition gegenüber den beteiligten Personen $[15,16,28,35]$. Wir konnten eine starke Tröpfchenbildung und somit Exposition des Operateurs anhand von 

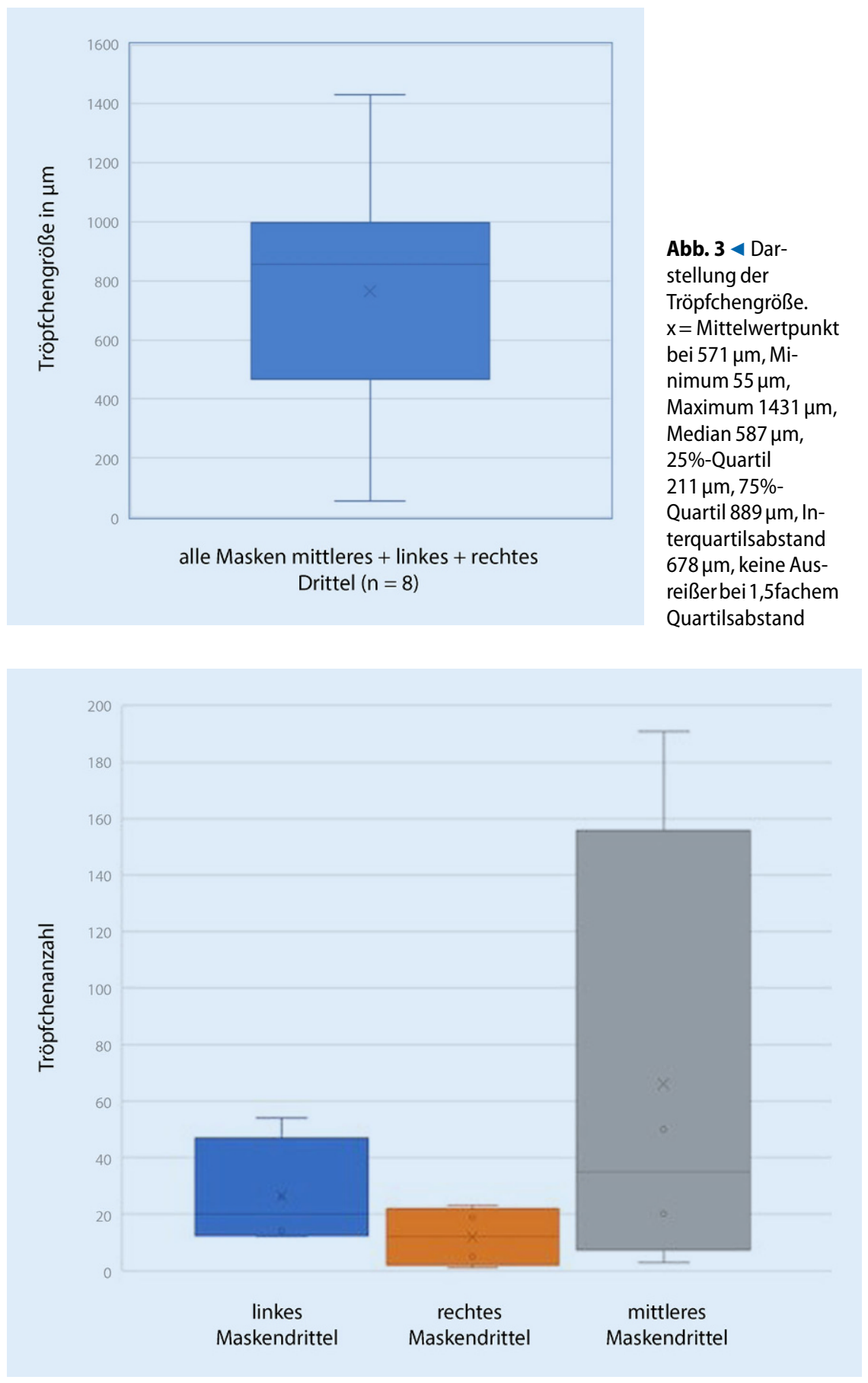

Abb. 4 \ Darstellung der Tröpfchenanzahl des linken, rechten und mittleren Drittels der Maske. Mittlere Anzahl der Tröpfchen des Gesichtsschilds: mittleres Drittel 29,13 Tröpfchen $\pm 37,15$; im rechten Drittel 4,38 Tröpfchen $\pm 5,70$; im linken Drittel 13,25 Tröpfchen \pm 9,34

Untersuchungen der Visiere der Operateure zeigen. Die Tröpfchengrößen variierten in ihrer Größe stark, sind aber eher als großvolumig anzusehen. Eine verhältnismäßig hohe Virusmenge ist deshalb im Fall von COVID-19-Patienten in den Tröpfchen wahrscheinlich. Hierfür spricht auch, dass neben Blutbestand- lich exponiert. Da alle Messungen oberhalb der Nasolabialfalte erfolgten, kann nur angenommen werden, dass auch der Mundbereich betroffen ist. Die persönliche Schutzausrüstung (PSA) kann, aus Sicht der Autoren, insofern nicht durch einfache Schutzbrillen in Kombination mit Halbmasken gewährleistet werden, sondern sollte durch eine frontale und laterale Ummantelung des Gesichts sichergestellt werden. Auch der Mund-und Nasenschutz sollte eine möglichst dichte und vollständige Ummantelung gewährleisten, da die Gefahr der Tröpfchenexposition bei nicht richtig anliegender Maske in die Atemwege des Operateurs ansonsten zu befürchten ist. Eine ausreichend sichere PSA zur Minimierung des Infektionsrisikos ist gerade im Umgang mit COVID-19-Patienten unumgänglich $[18,22]$. Auf Grundlage dieser Berichte und der Veröffentlichung einer aktuellen multidisziplinären Empfehlung von McGrath et al. [23] sollte eine PSA als Mindestanforderung aus einem FFP3-/N95-Mund-Nasen-Schutz, einem Schutz der Augen, einem flüssigkeitsabweisenden Einweg-Operationskittel und Handschuhen bestehen [30, 31]. Dabei ist nicht nur das Tragen der PSA, sondern auch das korrekte Anlegen und Ablegen der PSA ein wichtiger Faktor in der Vermeidung von Infektionen $[15,16,34,37]$.

Die Empfehlungen der Fachgesellschaften $[1,9,13,17]$, die auf der Grundlage von Beobachtungen der aktuellen COVID-19-Pandemie, aber auch der SARS-Pandemie von 2003 und 2004 sowie der MERS-Pandemie von 2007 entstanden sind, sehen hier in der Regel FFP2- oder FFP3-Masken vor, ggf. zusätzlich Schutzbrillen [3]. Die Klassifikation der verfügbaren filtrierenden Halbmasken (,filtering face piece“, FFP) wird nach europäischen Normen (DIN EN 149, - Tab. 3) vorgenommen [11]. Sie beschreibt die Gesamtleckage der Maske an den Undichtigkeitsstellen, die sich durch die Undichtigkeiten am Gesicht und Ausatemventil ergeben.

Das Tragen von Op.-Masken mit Gesichtsschild oder von Schutzbrillen schützt zusätzlich vor transokulären Infektionen durch Tröpfchen. Bei mutmaßlichem Risiko der transokulären Übertragung [40] ist demnach ein okulärer 


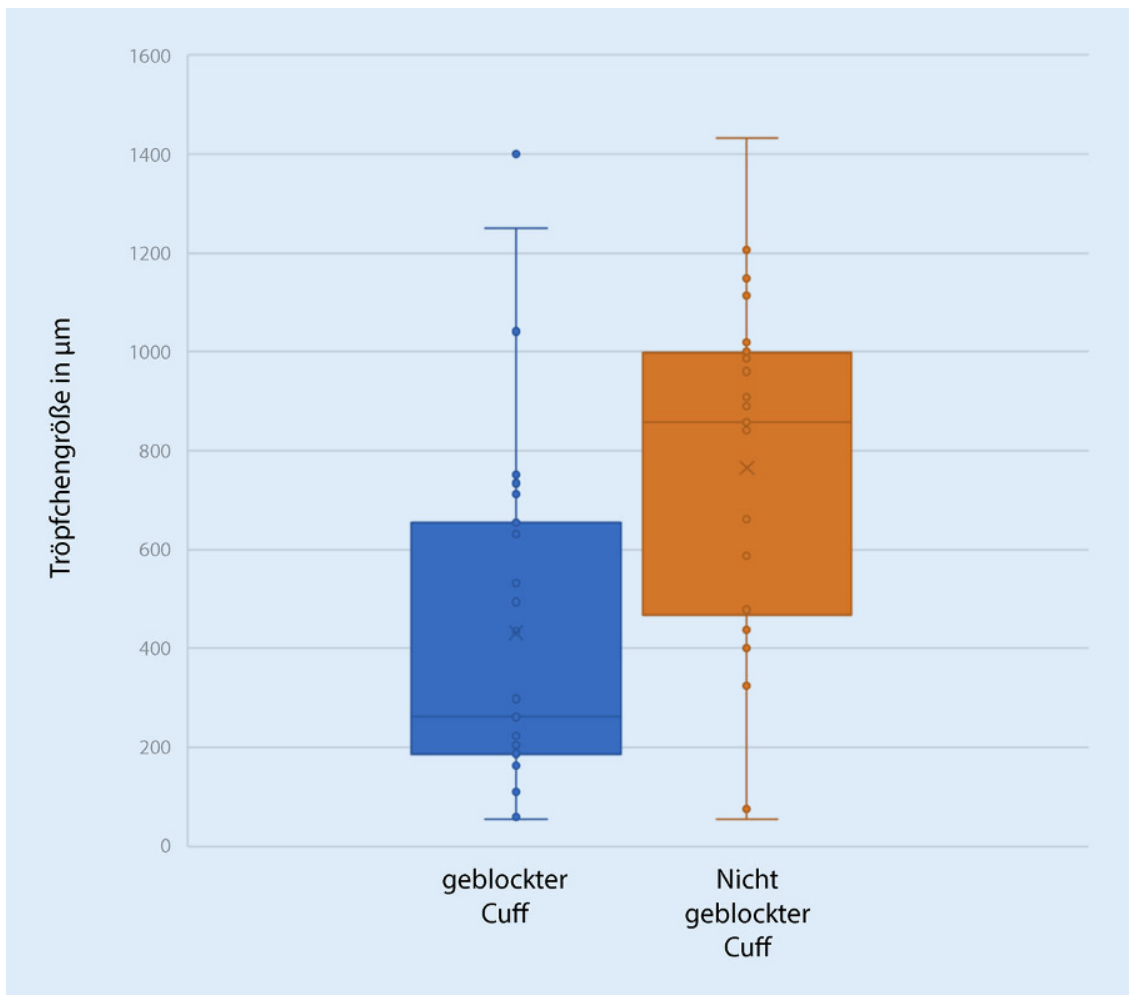

Abb. $5 \Delta$ Visiere der Patienten ( $n=2)$ mit nicht geblocktem Cuff (blau) und Patienten $(n=6)$ mit geblocktem Cuff (orange)

Schutz im Umgang mit COVID-19-Patienten essenziell. Sowohl die Verwendung von Gesichtsschilden als auch Schutzbrillen in Kombination mit Halbgesichtsmasken garantieren jedoch keinen umfassenden Schutz des Gesichts und seitlichen Kopfs. Die Tröpfchenexpositionsanalysen unserer Gesichtsschilde stellen jedoch auch in lateralen Gesichtsanteilen und der betrachteten Gesichtsfläche eine hohe Tröpfchenexposition dar, sodass auch eine Tröpfchenverteilung darüber hinaus denkbar ist. FFP2und FFP3-Halbmasken in Kombination mit einfachem okulärem Schutz bieten deshalb, aus Sicht der Autoren, einen lückenhaften Schutz und sind demnach für die Durchführung von Tracheotomien kein ausreichender Personenschutz bei hochinfektiösen Erkrankungen, die durch Tröpfchen übertragen werden. Die Einhaltung des kürzlich beschriebenen Verfahrens zur Tracheotomie mit Pausieren der Beatmung im Moment der Trachealeröffnung [28] reduziert die Tröpfchenexposition erheblich. Bei den in dieser Arbeit beschriebenen 3 Tracheotomien ohne Cuffruptur kam es artifiziellem Sekret ein Hustenstoß bei Intubation simuliert und die Tröpfchenausbreitung dargestellt. Tröpfchen waren auf dem Kittel, den Handschuhen, der Gesichtsmaske, dem „face shield“, aber auch an Haaren, Hals, Ohren und Schuhen des Untersuchers nachweisbar. AuBerdem kamen sie auf dem Boden in $1 \mathrm{~m}$ Entfernung und auf einem $2 \mathrm{~m}$ entfernten Monitor zur Darstellung. Die Tracheotomie bietet, gerade bei abrupt defektem Cuff, als Komplikation eines chirurgischen Eingriffs, eine vergleichbare Tröpfchenexposition zur Intubation. Erhöhte Sicherheitsmaßnahmen mit adäquatem Schutz des Personals sind demnach erforderlich [27]. Deshalb müssen auch die interdisziplinären Abläufe, wie An- und Ablegen der PSA, das Vorschieben des Tubus carinanah, das Stoppen der Beatmung während der Tracheaeröffnung und der Start der Beatmung gut kommuniziert und im Vorfeld trainiert werden. Pudszuhn et al. berichten auf der Grundlage eines standardisierten interdisziplinären und im Vorfeld trainierter Ablaufs (An- und Ablegen der PSA unter Buddy-Check, Vorschieben des Tubus carinanah, Pausieren der Beatmung kurz vor der Trachealeröffnung, gut kommunizierter Start der Beatmung), dass dieses Vorgehen zur Vermeidung der Bildung von Aerosolen und Tröpfchen beitragen kann [7, 28]. Dabei verweisen sie besonders auf die Wichtigkeit des Vorschiebens des Cuffs carinanah und auf das Sistieren der Beatmung während der Trachealeröffnung zur Vermeidung von Aerosolen und Tröpfchenbildung.

Kempfle et al. [16] beschreiben und analysieren das Management tracheotomierter Patienten. Dabei diskutieren die Autoren auch den umfassenden Schutz eines PAPR in Kombination mit einer Vollgesichts-Atemschutzmaske, die bei COVID-Tracheotomien in unserer Klinik auch zum Einsatz kommen. PAPR bestehen in der Regel aus einer Vollgesichtsmaske oder Haube, einer batteriebetriebenen Luftpumpe und einem Hochleistungsfilter. Bei diesen leistungsstarken luftreinigenden Atemschutzgeräten (PAPR) wird die Umgebungsluft vor dem Einatmen durch einen hocheffizienten HEPA-Filter („,high-efficiency particulate air") geführt. Hierdurch wird 


\begin{tabular}{llll}
\hline $\begin{array}{l}\text { Tab. 3 } \\
\text { Maske }\end{array}$ & $\begin{array}{l}\text { Filtering-Face-Piece-Masken nach DINEN 149 [11] } \\
\text { Gesamtleckage } \\
\text { (\%) }\end{array}$ & $\begin{array}{l}\text { Mittlerer Partikeldurch- } \\
\text { messer }\end{array}$ & Schutz vor \\
\hline FFP1 & $\leq 25$ & $0,6 \mu \mathrm{m}$ & $\begin{array}{l}\text { Tröpfchen/Aerosolen aus der } \\
\text { Umgebung }\end{array}$ \\
\hline FFP2 & $\leq 11$ & $0,6 \mu \mathrm{m}$ & $\begin{array}{l}\text { Tröpfchen/Aerosolen aus der } \\
\text { Umgebung }\end{array}$ \\
\hline FFP3 & $\leq 5$ & $0,6 \mu \mathrm{m}$ & $\begin{array}{l}\text { Tröpfchen/Aerosolen aus der } \\
\text { Umgebung }\end{array}$ \\
\hline $\begin{array}{l}\text { Mund-Nasen- } \\
\text { Schutz }\end{array}$ & $\geq 25$ & - & $\begin{array}{l}\text { Abgabe von Tröpfchen durch } \\
\text { den Träger }\end{array}$ \\
\hline
\end{tabular}

die Filtrationsleistung gegenüber FFPAtemschutzgeräten erhöht $[22,36]$, dies ist wichtig, da auch FFP3-Masken ein Aerosolinhalationsrisiko nicht vollständig ausschließen [16, 36]. Die PAPR umhüllt zumeist den gesamten Gesichtsschädel, schließt unter dem Op.-Mantel ab und bieten somit keine Eintrittspforte für die erregerhaltigen Tröpfchen [36]. Ein weiterer Vorteil ist, dass die Luftableitung dorsal, also patientenabgewandt erfolgt. Sie weisen darauf hin, dass je nach Modell ungereinigte Luft des Operateurs ein theoretisches Kontaminationsrisiko des sterilen Operationsfelds darstellen kann $[16,36]$, schränken dies allerdings modellbezogen ein und verweisen hierbei auf die zu diesem Thema nur begrenzten Daten hin. Die Vor- und Nachteile von PAPR, aber auch von anderen Maskentypen wurden im Rahmen der InfluenzaPandemie 2009 (H1N1) durch Tompkins und Kerchberger [36] umfassend dargestellt. Bei der Betrachtung der Vorzüge der PAPR legen sie für tröpfchen- und aerosolerzeugende Eingriffe die Verwendung eines „powered air-purifying respirator" (PAPR) nahe. Givi et al. ergänzen dies in Bezug auf die aktuelle Pandemie und weisen auf die Schwierigkeiten beim An- und Ausziehen und die damit verbundene Infektionsgefahr des medizinischen Personals, die Lautstärke der PAPR und damit verbundene eventuelle Verständnisschwierigkeiten explizit hin [32]. Alle Arbeiten verweisen ebenfalls auf die hohen Kosten und fehlende vergleichende Studien zu Infektionen von medizinischen Personal zwischen FFP3-/N95-Masken und Schutzbrille/Gesichtsschild. PAPR sind in den nationalen und internationalen Leitlinien und Empfehlungen zur Tracheotomie [1, 9, 12] nicht explizit empfohlen, ihr hoher Infektionsschutz wird aber herausgestellt.

Unsere Pilotstudie unterstützt die Empfehlungen der nationalen und internationalen HNO-Fachgesellschaften (Deutsche Gesellschaft für Hals-NasenOhren-Heilkunde, Kopf- und Hals-Chirurgie e.V., DGHNO-KHC; American Academy of Otolaryngology-Head and Neck Surgery, AAO-HNS; Ear, Nose and Throat surgery United Kingdom, ENTUK) im Umgang mit SARS-CoV-2 [1, 9, 12]. Hiernach werden übereinstimmend bei Vorliegen oder Verdacht auf eine COVID-19-Erkrankung mindestens eine FFP2-Schutzmaske in Kombination mit einem okulären Schutz bei Untersuchungen und Operationen der oberen Atemwege sowie ein erweiterter Schutz bei besonderer Exposition, wie er bei der Durchführung einer Tracheotomie vorliegt, empfohlen.

\section{Fazit für die Praxis}

In dieser Studie wurde eine ausgeprägte Tröpfchenexposition des medizinischen Personals bei Tracheotomien aufgezeigt. Bei infektiösen Patienten, aber insbesondere bei COVID-19-Patienten, sollten maximale Ansprüche an die persönliche Schutzausrüstung gestellt werden.

\section{Korrespondenzadresse}

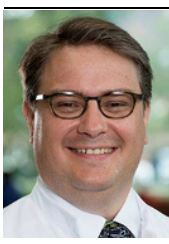

Dr. med. C. Plettenberg

Hals-Nasen-Ohrenklinik, Zentrum für Operative Medizin II, Universitätsklinikum Düsseldorf

Moorenstr. 5, 40225 Düsseldorf, Deutschland christian.plettenberg@ med.uni-duesseldorf.de
Funding. Open Access funding enabled and organized by Projekt DEAL.

\section{Einhaltung ethischer Richtlinien}

Interessenkonflikt. C. Plettenberg, K. Geipel, I. Stenin, T. Klenzner, M. Wagenmann, J. Schipper und K. Scheckenbach geben an, dass kein Interessenkonflikt besteht.

Alle beschriebenen Untersuchungen wurden in Kooperation mit der zuständigen Ethikkommission, im Einklang mit nationalem Recht sowie gemäß der Deklaration von Helsinki von 1975 (in der aktuellen, überarbeiteten Fassung) durchgeführt. Für die aufgeführten Studien gelten die jeweils dort angegebenen ethischen Richtlinien.

Open Access. Dieser Artikel wird unter der Creative Commons Namensnennung 4.0 International Lizenz veröffentlicht, welche die Nutzung, Vervielfältigung, Bearbeitung, Verbreitung und Wiedergabe in jeglichem Medium und Format erlaubt, sofern Sie den/die ursprünglichen Autor(en) und die Quelle ordnungsgemäß nennen, einen Link zur Creative Commons Lizenz beifügen und angeben, ob Änderungen vorgenommen wurden.

Die in diesem Artikel enthaltenen Bilder und sonstiges Drittmaterial unterliegen ebenfalls der genannten Creative Commons Lizenz, sofern sich aus der Abbildungslegende nichts anderes ergibt. Sofern das betreffende Material nicht unter der genannten Creative Commons Lizenz steht und die betreffende Handlung nicht nach gesetzlichen Vorschriften erlaubt ist, ist für die oben aufgeführten Weiterverwendungen des Materials die Einwilligung des jeweiligen Rechteinhabers einzuholen.

Weitere Details zur Lizenz entnehmen Sie bitte der Lizenzinformation auf http://creativecommons.org/ licenses/by/4.0/deed.de.

\section{Literatur}

1. AAO-HNS American Academy of OtolaryngologyHead and Neck Surgery https://www.entnet.org/ content/coronavirus-disease-2019-resources. Zugegriffen: 9.8.2020

2. AWMF Leitlinie (2016) Hygieneanforderungen bei ausgewählten respiratorisch übertragbaren Infektions-Erkrankungen (aerogen und Tröpfchen), 5. Aufl. (Langversion, Stand 01/2016)

3. AWMF Leitlinie (2017) S3-Leitlinie Invasive Beatmung und Einsatz extrakorporaler Verfahren bei akuter respiratorischer Insuffizienz, 1. Aufl. (Langversion, Stand 04.12.2017)

4. Bishop MJ, Hibbard AJ, Fink BR (1985) Laryngeal injury in a dog model of prolonged endotracheal intubation. Anesthesiology 62(6):770-773

5. Bunyan D, Ritchie L, Jenkins D, Coia JE (2013) Respiratory and facial protection: a critical review of recent literature. JHosp Infect 85:165-169

6. Canelli R, Connor CW, Gonzalez M, Nozari A, Ortega $R$ (2020) Barrier enclosure during endotracheal intubation. NEng J Med 382:1957-1958

7. Chughtai AA, Seale $H$, Islam MS, Owais $M$, Macintyre CR (2020) Policies on the use of respiratory protection for hospital health workers 
to protect from coronavirus disease (COVID-19). Int J Nurs Stud 105:103567

8. Davis K Jr., Campbell RS, Johannigman JA, Valente JF, Branson RD (1999) Changes in respiratory mechanics after tracheostomy. Arch Surg 134(1):59-62

9. Deutsche Gesellschaft für HNO-Heilkunde, Kopfund Hals-Chirurgie (DGHNO-KHC) und ihrer Arbeitsgemeinschaft Laryngologie und Trachealerkrankungen Chirurgische Aspekte zur Tracheostomie bei Covid-19 positiven Patienten. https://cdn.hno.org/media/CoronaTicker/ SN_Chirurg_Aspekte_Tracheostomie_Covid-19. pdf.Zugegriffen: 8.9.2020

10. Diehl JL, el Atrous S, Touchard D, Lemaire F, Brochard L (1999) Changes in the work of breathing induced by tracheotomy in ventilator-dependent patients. Am J Respir Crit Care Med 159(2):383-388

11. DIN EN 149:2009-08 (2009) Atemschutzgeräte Filtrierende Halbmasken zum Schutz gegen Partikeln - Anforderungen, Prüfung, Kennzeichnung. Beuth, Berlin

12. Doremalen N, Morris DH, Holbrook MG, Gamble A, Williamson BN, Tamin A, Harcourt JL, Thornburg NJ, Gerber SI, Lloyd-Smith JO, de Wit E, Munster VJ (2020) Aerosol and surface stability of SARS-COV-2 as compared with SARS-COV-1. N Engl J Med 382:1564-1567

13. ENT-UK https://www.entuk.org/sites/default/ files/files/COVID\%20tracheostomy\%20guidance compressed.pdf.Zugegriffen: 8.9.2020

14. Fernstrom A, Goldblatt M (2013) Aerobiology and its role in the transmission of infectious diseases. JPathog 2013:493960

15. Givi B, Schiff BA, Chinn SB, Clayburgh D, lyer G, Jalisi S, Moore MG, Natha CA, Orloff LA, O'Neill JP, Parker N, Zender C, Morris LGT, Davies L (2020) Safety recommendations for evaluation and surgery of the head and neck during the COVID19 pandemic. JAMA Otolaryngol Head Neck Surg 146(6):579-584

16. Kempfle JS, Löwenheim H, Huebner MJ, Müller SK (2020) Management von Patienten mit Tracheostoma während der COVID-19-Pandemie: Literaturüberblick und Demonstration. HNO 68:828-837

17. Kluge $S$, Janssens $U$, Welte $T$, Weber-Carstens $S$, Schälte G, Salzberger B, Gastmeier P, Langer F, Wepler M, Westhoff M, Pfeifer M, Hoffmann F, Böttiger BW, Marx G, Karagiannidis C (2020) Empfehlungen zur intensivmedizinischen Therapie von Patienten mit COVID-19 (AWMF S1-Leitlinie Stand 21.07.2020 (Version 3))

18. Kwan A, Fok WG, Law Kl, Lam SH (2004) Tracheostomy in a patient with severe acute respiratory syndrome. Br J Anaesth 92:280-282

19. Li Q, Guan X, Wu P, Wang X, Zhou L, Tong Y, Ren R, Leung KSM, Lau EHY, Wong JY, Xing $X$, Xiang $N$, Wu Y, Li C, Chen Q, Li D, Liu T, Zhao J, Liu M, Tu W, Chen C, Jin L, Yang R, Wang Q, Zhou S, Wang R, Liu H, Luo Y, Liu Y, Shao G, Li H, Tao Z, Yang Y, Deng Z, Liu B, Ma Z, Zhang Y, Shi G, Lam TTY, Wu JT, Gao GF, Cowling BJ, Yang B, Leung GM, Feng Z (2020) Early transmission dynamics in Wuhan, China, of novel Coronavirus-infected pneumonia. N Engl J Med 382(13):1199-1207

20. Liu J, Zheng X, Tong Q, Li W, Wang B, Sutter $\mathrm{K}$, Trilling M, Lu M, Dittmer U, Yang D (2020) Overlapping and discrete aspects of the pathology and pathogenesis of the emerging human pathogenic coronaviruses SARS-CoV, MERS-CoV, and 2019-nCoV.J Med Virol 92(5):491-494

21. Loeb M, Dafoe N, Mahony J, John M, Sarabia A, Glavin V, Webby R, Smieja M, Earn DJ, Chong S,
Webb A, Walter SD (2009) Surgical mask vs N95 respiratorfor preventing influenza among health care workers: a randomized trial. JAMA 302(17):1865-1871

22. Lu CW, LiuXF, Jia ZF (2020) 2019-nCoV transmission through the ocular surface must not be ignored. Lancet 395(10224):e39

23. McGrath BA, Brenner MJ, Warrillow SJ, Pandian V, Arora A, Cameron TS, Añon JM, Martínez GH, Truog RD, Block SD, Lui GCY, McDonald C, RassekhCH,Atkins J, Qiang L, VergezS, DulguerovP, Zenk J, Antonelli M, Pelosi P, Walsh BK, Ward E, Shang $Y$, Gasparini $S$, Donati A, Singer $M$, Openshaw PJM, Tolley N, Markel H, FellerKopman DJ (2020) Tracheostomy in the COVID-19 era: global and multidisciplinary guidance. Lancet Respir Med 8(7):717-725

24. Maclntyre $C R$, Wang $Q$, Cauchemez $S$, Seale $H$, Dwyer DE, Yang P, Shi W, Gao Z, Pang X, Zhang Y, Wang X, Duan W, Rahman B, Ferguson N (2011) A cluster randomizedclinical trial comparing fit-tested and non-fit-tested N95 respiratorsto medical masks to prevent respiratory virus infection in healthcare workers. Influenza Other Respir Viruses 5(3):170-179

25. Ng K, Poon BH, Kiat Puar TH et al (2020) COVID-19 and the risk to health care workers: a case report Ann Intern Med 172(11):766-767

26. Nordin U (1977) The trachea and cuff-induced tracheal injury. An experimental study on causative factors and prevention. Acta Otolaryngol Suppl 345:1-71

27. Pozuelo-Carrascosa DP, Herráiz-Adillo Á, AlvarezBueno C, Añón JM, Martínez-Vizcaíno V, CaveroRedondo I (2020) Subglottic secretion drainage for preventing ventilator-associated pneumonia: an overview of systematic reviews and an updated meta-analysis. Eur Respir Rev 29(155):12

28. Pudszuhn A, Voegeler S, Berger $C$, Treskatsch $S$, Angermair S, Hansen S, Hofmann VM (2020) Elektive Tracheostomie bei COVID-19-Patienten - Erfahrungen mit einem standardisierten interdisziplinären Vorgehen. HNO68(11):838-846

29. Rello J, Soñora R, Jubert $P$, Artigas Rué Vallés AMJ (1996) Pneumonia in intubated patients. Role of respiratory airway care. Am J Respir Crit Care Med 154(1):111-115

30. Repici A, Maselli R, Colombo M, Gabbiadini $R$, Spadaccini M, Anderloni A, Carrara S, Fugazza A, Di Leo M, Galtieri PA, Pellegatta G, Ferrara EC, Azzolini E, Lagioia M (2020) Coronavirus (COVID19) outbreak: what the department of endoscopy should know. Gastrointest Endosc 92(1):192-197

31. Robert-Koch-Institut https://rki.de/DE/ Content/InfAZ/Neuartiges_Coronavirus/PSA Fachpersonal/Dokumente_Tab.html.Zugegriffen: 21.9.2020

32. Seymour CW, Martinez A, Christie JD, Fuchs PD (2004) The outcome of extubation failure in a community hospital intensive care unit: a cohort study. Crit Care 8(5):R322-R327

33. Shapiro M, Wilson RK, Casar G, Bloom K, Teague RB (1986) Work of breathing through different sized endotracheal tubes. Crit Care Med 14(12):1028-1031

34. Tay JK, Khoo ML, Loh WS (2020) Surgical considerations for tracheostomy during the COVID-19 pandemic: lessons learned from the severeacute respiratory syndrome outbreak.JAMA 146(6):517

35. Thamboo A, Lea J, Sommer DD, Sowerby $L$, Abdalkhani A, Diamaond C, Ham J, Hefferman A, Long MC, Phulka J, Wu YQ, Yeung P, Lammers M (2020) Clinical evidence based review and recommendations of aerosol generating medical procedures in otolaryngology- Head and neck surgery during the COVID-19 pandemic. JOtolaryngol Head Neck Surg 49(1):28

36. Tompkins BM, Kerchberger JP (2010) Special article: personal protectiveequipment for care of pandemic influenza patients: a training workshop for the powered air purifying respirator. Anesth Analg 111:933-945

37. Wang D, Hu B, Hu C et al (2020) Clinical characteristics of 138 hospitalized patients with 2019 novel coronavirus-infected pneumonia in Wuhan, China. JAMA 323(11):1061-1069

38. Wei WI, Tuen HH, Ng RW, Lam LK (2003) Safe tracheostomy for patients with severe acute respiratory syndrome. Laryngoscope 113(10):177-179

39. Wölfel R, Corman VM, Guggemos W, Seilmaier M, Zange S, Müller MA, Niemeyer D, Jones TC, Vollmar P, Rothe C, Hoelscher M, Bleicker T, BrüninkS, Schneider J, Ehmann R, Zwirglmaier K, Drosten C, Wendtner C (2020) Virological assessment of hospitalized patients with COVID-2019. Nature 581(7809):465-469

40. World Health Organisation (2020) Transmission of SARS-CoV-2: implications for infection prevention precautions. https://www.who. int/publications/i/item/modes-of-transmissionof-virus-causing-covid-19-implications-for-ipcprecaution-recommendations. Zugegriffen: 21.9.2020 